\title{
THE ULTIMATE RADIO RESOLUTION
}

\author{
N.S.KARDASHEV \\ Astro Space Center of P.N.Lebedev Physical Institute \\ Leninsky Pr. 53, Moscow, 117924, Russia \\ L. I. GURVITS* \\ National Astronomy and Ionosphere Cienter \\ A recibo Observatory, P.O.Box 995, Arecibo, Puerto Rico 00613 \\ and \\ G.S.TSAREVSKY \\ Astro Space Center of P.N.Lebedev Physical Institute \\ Leninsky Pr. 53, Moscow, 117924, Russia
}

\begin{abstract}
Scientific objectives and some technical issues of Space VLBI (SVLBI) are considered. Two SVLBI missions under preparation - RadioAstron and VSOP - are briefly described.

Key words: VLBI - RadioAstron - VSOP
\end{abstract}

\section{Introduction}

The simple but fundamental formula $\theta=\lambda / D$ ( $\lambda$ is the wavelength, $D$ is the diameter of a single- or the baseline length of a multi-element aperture, and $\theta$ is the angular resolution) leaves only one choice to decrease $\theta$ within given range of $\lambda$. This way is to increase $d$. During the last few decades very wide range of baselines from hundreds meters up to Very Long Baselines Interferometers (VLBI) dealing with baselines up to tens thousands kilometers have been opened up in radio wavelength range. Due to its record angular resolution VLBI technique occupies a special place among other possible tools for study of the Universe (Fig. 1). The absolutely genuine continuation of this technique development is nothing else but increasing baselines beyond the Earth diameter.

\section{How Long Can Be Very Long Baselines?}

There are two principle reasons which can limit increasing of baselines:

1. The nature of astrophysical objects which might be not compact and bright enough to be detected with baselines longer than reached ones to the moment.

2. Propagation phenomena distorting radio images of sources.

To reformulate the first reason quantitatively let us consider a simple energetic formula describing the spectral density of radiation flux from a source. Let us express this flux density in two forms:

$$
F_{\nu}=2 k T_{B} \Omega \lambda^{-2} \quad \text { and } \quad F_{\nu}=2 k \alpha T_{N}\left(2 A_{1} A_{2} \Delta \nu \Delta t\right)^{-0.5}
$$

The first form describes flux density $F_{\nu}$ from a source of brightness temperature $T_{B}$ at the wavelength $\lambda$ which has the solid angle $\Omega$ ( $k$ is the Boltzmann constant).

* On leave from Astro Space Center of P.N.Lebedev Physical Institute, Leninsky Pr. 53, Moscow, 117924, Russia 


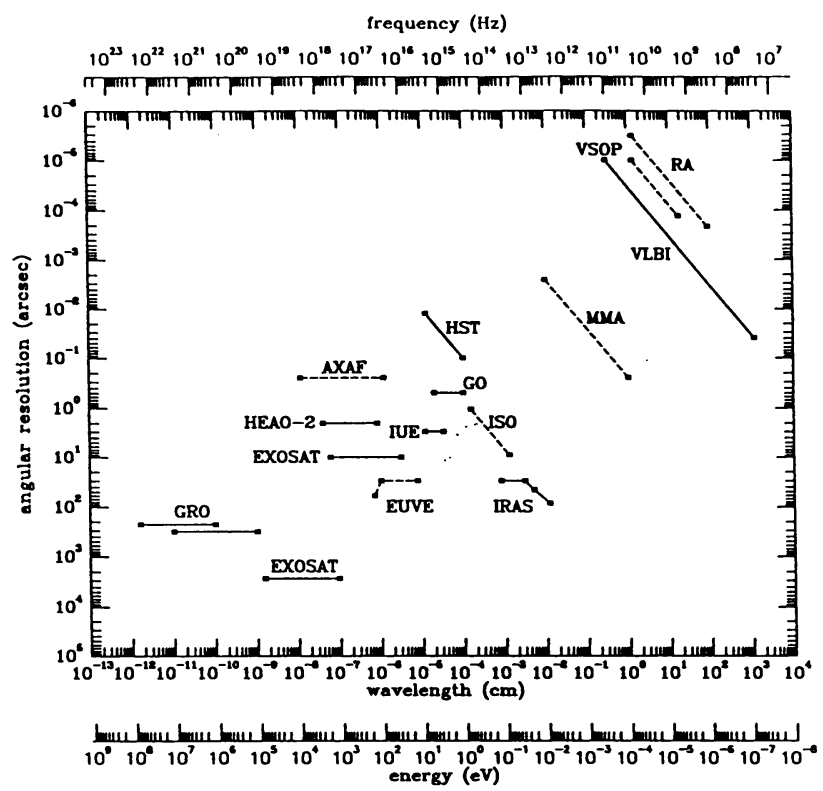

Fig. 1. The best indices of angular resolution for all available wavelengths of electromagnetic spectrum. Solid lines show available facilities or completed missions, dashed projects under preparation. Abbreviations of instruments are: AXAF - Advanced X-ray Astronomy Facility; EUVE - Extreme Ultraviolete Explorer; EXOSAT - X-ray Astronomical Satellite; GRO - Gamma Ray Observatory; GO - Ground optical telescopes; HEAO-2 - High Energy Astrophysics Observatory (Einstein); HST - Hubble Space Telescope; IRAS - Infrared Astronomical Satellite; ISO - Infrared Space Observatory; IUE International Ultraviolete Explorer; MMA - Millimeter Array; RA - RadioAstron; VLBI - Ground VLBI; VSOP - VLBI Space Observatory Program.

The second form corresponds to the response of a two element interferometer, where $T_{N}$ - the system noise temperature, $A_{1}$ and $A_{2}$ - collecting areas of interferometer elements, $\Delta \nu$ is the bandwidth, and $\Delta t$ is the integration time. The coefficient $\alpha$ is a product of all specific hardware dependent coefficients among which the most significant one is the signal-to-noise ratio (all others can be considered by order of units). Using these two forms and assuming the best fit between the source structure and the instrumental pattern one may derive an estimating formula for the maximal baseline $B_{\max }$ for observation of a source with the given signal-to-noise ratio:

$$
\frac{B_{\max }}{10^{5} \mathrm{~km}} \cong\left[\frac{\Delta \nu}{64 \mathrm{MHz}} \cdot \frac{\Delta t}{100 \mathrm{~s}}\right]^{0.25}\left[\frac{D_{1}}{100 \mathrm{~m}} \cdot \frac{D_{2}}{10 \mathrm{~m}}\right]^{0.5}\left[\frac{T_{B}}{10^{12} \mathrm{~K}}\right]\left[\frac{T_{N}}{100 \mathrm{~K}} \cdot \frac{\alpha}{100}\right]^{-0.5}
$$

The "technical" parameters (i.e. all except $T_{B}$ ) used in the right part of this estimation are typical for the current and nearest future state of VLBI technique. So, taking a "standard" limit of brightness temperature $T_{B}=10^{12} \mathrm{~K}$ for incoherent synchrotron sources (Kellermann and Pauliny-Toth, 1969) one may expect to detect a source at baselines up to $\sim 10^{5} \mathrm{~km}$. 
Few known mechanisms of emission can provide in principle much higher brightness temperature. Such a mechanism could be realized in the pulsar magnetosphere, where brightness temperature could be as high as $10^{30} \mathrm{~K}$. Application of VLBI technique for pulsar investigations might acquire especial significance after recent discovery of planets around the pulsar 1257+12 (Wolszczan, Frail, 1992). Another possibility for some exceeding of the mentioned limit could be achieved at narrow spectral windows for some spectral lines.

Some evidences of exceeding of $10^{12} \mathrm{~K}$ limit were obtained for the classical case of a continuum non-thermal sources. Temperatures $4 \times 10^{12} \mathrm{~K}$ were directly detected in the TDRSS-OVLBI experiment (Levy et al., 1986). Quirrenbach et al. (1989) estimated the brightness temperature up to $10^{18}-10^{19} \mathrm{~K}$ from observations of variability of extragalactic radio sources. Possible explanations of this phenomenon are discussed from various points of view by Shapirovskaya (1991) and Slysh (1992).

The second principle limit is caused by a number of propagation media, such as atmosphere, ionosphere, interplanetary, interstellar, and intergalactic media, and proper envelope of a source (the last medium could be refered among issues of the first reason, though). Not discussing details let us mention only the strong evidence to get certain VLBI fringes at $\mathrm{cm}$-dm wavelengths for baselines up to $10^{5}$ $\mathrm{km}$ (Shishov, 1993). Much stronger statement could be done from the detection of transient fringes in so called interstellar interferometer at effective baselines $\sim 1$ A.U. (Wolszczan, Cordes, 1987). One can conclude from this result that at least sometimes in some directions there are conditions for successful observation at baselines few order of magnitude longer than $10^{5} \mathrm{~km}$.

\section{Scientific Objectives For Space VLBI}

3.1. Cosmology. One of the most fundamental problem of the astronomy - measuring of distances - could be solved using SVLBI technique on the new level of accuracy. In the "small" scale (in cosmological terms) such an advantage is connected with a higher resolution measurement of proper motion of $\mathrm{H}_{2} \mathrm{O}$ maser spots. An extrapolation of the ground VLBI result of Reid et al. (1988) concerning the source $\operatorname{Sgr} B 2(N)$ on extragalactic scales using about 10 times higher angular resolution indicates a principle possibility to measure directly distances up to $10 \mathrm{Mpc}$.

Observations of AGN will realize advantages of SVLBI at longèr distances. As was shown (see Pelletier and Roland (1989) and references therein) cosmological parameters could be effectively estimated from measurements of proper motion in AGN structures. Direct measurements of apparent angular sizes of AGN also are shown as an effective approach for estimation of cosmological parameters (Kellermann, 1993). Even measurements of a visibility amplitude in few $u v$-points without imaging can provide very valuable information for cosmological applications (Gurvits, 1993).

The most probable candidate for gravitational lensed image $0957+561$ shows the obvious necessity to go down 1 milliarcsecond resolution to provide a critical test of lensed nature. The analysis of sub-milliarcsecond structure of lensed objects will give a possibility to construct an adequate model of lensing object and hence to "weight" it (Turner, 1988). It applies directly to the "hidden mass" problem. 
3.2. Discrete Continuum Sources. This class of targets is presented mostly by AGN on extragalactic scales. Assuming $10^{5} \mathrm{~km}$ as a baseline of SVLBI we will obtain typical angular resolution of tens $\mu a s$ at $\mathrm{cm}$-wavelength domain. It gives a linear resolution better than $10^{16} \mathrm{~cm}$ or about 3 light days for the nearest AGNs (as Cen $A, M 81)$. some special interest is connected with high redshift sources, since $T_{B} \sim[\gamma /(1+z)]^{3+\alpha}$, where $\gamma$ is the Lorentz factor, $\alpha$ - the spectral index.

Again as was noted above, not only imaging SVLBI experiments could be useful for studies of galaxy nuclei. Even a detection of fringes at baselines much longer than the Earth diameter would be of great interest for estimation of sizes and hence physical conditions in the emitting regions.

Super novae and their remnants as targets for future SVLBI observations also should be referenced as objects of a certain interest.

Stars became objects of VLBI studies quite recently and they remain rather difficult targets for VLBI observations since they are too weak radio sources. Some progress is achieved during last few years with using the most sensitive wide band VLBI system with largest ground radio telescopes for observations of few particular stars (Taylor, 1993). The first generation of SVLBI missions will give some exploratory view on sub-mas structure of the most strong radio stars.

3.3. Spectral Line VLBI. One of the most popular lines studied with VLBI, the $\mathrm{OH}$ line $18 \mathrm{~cm}$, will remain as a target only at relatively low orbits (up to units of the Earth diameter) due to significant broadening of images in the interstellar medium.

SVLBI future of the $\mathrm{H}_{2} \mathrm{O}$ line $1.35 \mathrm{~cm}$ looks more optimistic. One class of this emission sources is related with bright hot spots in some HII regions. The corresponding brightness temperature can reach $10^{15} \mathrm{~K}$. An estimated linear size of such sources is in the range of $0.1 \div 10 \mathrm{~A}$.U. and in many cases is lower than the angular resolution of ground VLBI. Another class of $\mathrm{H}_{2} \mathrm{O}$ maser sources is presented by Herbig-Haro objects. Higher angular resolution is subject of crucial interest for investigations of these objects.

Some further plans might imply studies of other lines (as $\mathrm{SiO}, \mathrm{CO}$ etc.).

3.4. Astrometry and Geodesy. Ground VLBI experiments provide two orders of magnitude more accurate measurements of co-ordinates of celestial sources than any other technique. Quite impressive results on measurements of intercontinental distances with centimeter accuracy also should be mentioned as an undoubtful success of ground VLBI. In principle, SVLBI would be able to push forward these results. Such experiments require higher than "imaging" experiments accuracy of a-priori knowledge of telescopes positions. The first generation of SVLBI missions will be able to check some elements of astrometric and geodetic applications. Future generation of SVLBI missions will be able to utilize these applications very fruitfully. In particular, two missions working simultaneously, as shown by Alekseev (1993), might give new qualitative improvement of the resulting accuracy of astrometric measurements.

3.5. SVLBI and SETI. One of the most reliable criteria of the artificial nature of the cosmic signal is the compactness of the signal source. Due to obvious reasons VLBI in general, and SVLBI as its advanced case can provide the best application of this criterion for a hypothetical artificial extraterrestrial signal. 


\section{SVLBI as a Catalyst of New Experimental Methods}

4.1. The use of spatial redundancy (Noordam, de Bruyn, 1982) is stricted by fixed geodetical positions and hence synchronous motion in the inertial frame all elements of the array. This is not a case for a space radio telescope. Such a motion can provide more spatial redundancy (Gurvits and Pogrebenko, 1985).

4.2. Wide range (multi-frequency) VLBI. The bandwidth in VLBI experiments usually is a small fraction (normally about or less than $1 \div 3 \%$ ) of a sky frequency. This allows to avoid some non-linear effects stipulated by both physics of the source and properties of a data flow path. However wider band may provide some advantages by increasing of the signal-to-noise ratio and improving the $u v$-plane coverage. The idea was considered conformably to SVLBI by Andreyanov et al. (1982). Some successful experiments with multi-frequency synthesis were carried out using ground VLBI (Conway et al., 1990).

4.3. Burst sampling was firstly proposed for ground mm-wavelength VLBI (Kawaguchi, 1991). Technically the method needs hardware which is similar to one under preparation for VSOP project. In this sense SVLBI and mm-VLBI techniques supports each other and reciprocally supply by commonly required instrumentation.

4.4. Precise baseline determination was already mentioned as allowing to use VLBI observations for astrometry and geodesy. The same property may be used as a feedback for any experiments were extremely precise measurements of long distances, including extra-terrestrial, are required. So, SVLBI may provide a very effective way to search for gravitational waves (Braginsky et al., 1992).

\section{Space VLBI Projects: Status and the Nearest Plans}

The idea to put a radio telescope into the orbit was discussed since the initial steps of VLBI in middle $60 \mathrm{~s}$. The first space-borne $10-\mathrm{m}$ antenna KRT-10 was tested on-board "Salyut-6" orbital station in 1979. The first light from space VLBI is obtained by the TDRSS-OVLBI experiment which gives a brilliant example of an ad-hoc experiment (Levy et al., 1986). Two other projects have been investigated extensively by international teams of experts created by European Space Agency. These projects are QUASAT (first half of 80s, see Schilizzi, 1988) and IVS (198991, see Pilbratt, 1991). Both projects failed due to funding problems but brought in some meaningful thoughts and results for future development of the subject.

The current status of SVLBI is connected with two approved missions, both scheduled for middle 90 s. These missions are RadioAstron (the leading institutions - Astro Space Center of P.N.Lebedev Physical Institute and Lavochkin Association, Russia), and VSOP (the leading institution - Institute for Space and Astronautical Science, Japan). The general description of the missions are given by Kardashev and Slysh (1988) and Nishimura (1991) correspondingly, and some their parameters are summarized in Table 1. Both missions are developing in close coordination. It allows to investigate some common problems, specially operational and data acquisition issues, maximizing the scientific output of both missions.

5.1. The RadioAstron project implies creating an orbiting radio telescope onboard the spacecraft "Spectr-R" - one of several astrophysical missions of "Spectr" 
TABLE I

RadioAstron and VSOP specification

\begin{tabular}{||lcc||}
\hline \hline & RadioAsrtron & VSOP \\
\hline Inclination & $51.6^{\circ}$ & $31^{\circ}$ \\
Orbital period & 28 hours & 6 hours \\
Apogee altitude & $79,000 \mathrm{~km}$ & $20,000 \mathrm{~km}$ \\
Perigee altitude & $2,000 \mathrm{~km}$ & $1,000 \mathrm{~km}$ \\
Argument of perigee & $300^{\circ} \pm 20^{\circ}$ & \\
Total mass & $4,950 \mathrm{~kg}$ & $800 \mathrm{~kg}$ \\
Antenna diameter & $10 \mathrm{~m}$ & up to $10 \mathrm{~m}$ \\
Frequencies & $0.327,1.6,4.9,22 \mathrm{GHz}$ & $1.6,4.9,22 \mathrm{GHz}$ \\
Data acquisition & VLBA and S2 & K4 and VLBA \\
\hline \hline
\end{tabular}

series. "Spectr-R" will be launched with "Proton" launcher on high-excentricity near-one day orbit. Such a period provides a maximal baseline about $80,000 \mathrm{~km}$.

The main feature of the RadioAstron design is the $10-\mathrm{m}$ parabolic antenna. Its mass is about $1500 \mathrm{~kg}$. It will be launched in folded state and then will be deployed in the orbit. The reflector surface is formed by 27 solid petals and the central 3$\mathrm{m}$ diameter part. The antenna petals are produced from carbon-fiber composite material which provides high solidity under strong weight and thermal deformation requirements. The rms accuracy of the surface is expected to be within $0.5 \mathrm{~mm}$.

RadioAstron will have the simplest optical scheme with feeds in the prime focus of the main reflector. Such a scheme allows to avoid some extra weight and design problems comparably with two or more reflectors scheme. But at the same time this single reflector scheme put some specifical requirements for feeds design and operational logics. In particular, it requires either different offsets of the main reflector pointing for different frequencies or a special co-axial feed design. The latest, rather unusual for ground radio astronomical custom option is chosen for the RadioAstron. A focal package with feeds, low noise amplifiers and radio astronomical receivers is attached to the inner 3-m diameter part of the telescope by six bearing.

RadioAstronomical receivers will be placed inside the focal package. They will operate in both circular polarizations at all four frequencies. The bandwidth in each polarization at all frequencies but $327 \mathrm{MHz}$ will be $32 \mathrm{MHz}$. Hence, the maximal signal band (the sum of two polarization channels) will be $64 \mathrm{MHz}$ at three higher frequencies. (This is the value of $\Delta \nu$ used for the estimation in the Section 2). The analogous numbers for $327 \mathrm{MHz}$ channel will be 4 and $8 \mathrm{MHz}$ respectively.

Another basic part of VLBI data flow equipment will be placed inside the service module - the main body of the spacecraft. The standard $500 \mathrm{MHz}$ IF signal will come to video convertors and than digitized and formatted. The output of formatter will be connected with data down-link system with two $32 \mathrm{MHz}$ channels.

The orbital parameters shown in the Table 1 are initial ones. During the lifetime of the mission - 3 years - they will evolve due to gravitational influence from the Sun, the Moon, non-spherical type of the Earth gravitational field, aerodynamical 
influences, light pressure and some other less significant disturbances.

RadioAstron data recorded with VLBA data acquisition system will be processed at the VLBA Data Processing Center at NRAO (Socorro, NM, USA). The S2 data will be processed by correlators designed at the Dominion Radioastrophysical Observatory and Institute of Space and Terrestrial Sciences (Canada).

The RadioAstron project is been developing by joint efforts of experts from Australia, Canada, Finland, Germany, Hungary, India, Italy, Kazakhstan, The Netherlands, Russia, Sweden, Ukraine, United Kingdom, USA, Uzbekistan.

5.2. VSOP (VLBI Space Observatory Program) is proposed by scientists from number of Japanese institutes in middle $80 \mathrm{~s}$. The project foresees to put satellite "Muses-B" onto 6-hrs orbit using the new launcher M-V.

Due to very strong weight budget limitations designers of the antenna have chosen an original tension-activated truss design of the main reflector. Such a design provides very compact packing of the antenna under the head fairing of the launcher and very small mass of the antenna. The expected surface accuracy is $0.5 \mathrm{~mm}$. This will give possibility to operate at the highest frequency $22 \mathrm{GHz}$ with reasonable efficiency. All observations will be carried out in the left circular polarization.

The optical scheme of the radio telescope is two-reflecting with the secondary focus placed near the main body of the spacecraft. It gives possibility to have only one container (the main body) for all kind of on-board equipment.

The prime scientific aim of the VSOP mission is the high imaging capability. This requires resonably complete $u v$-plane coverage, and hence not too high orbit. The compromise between two desires - to have both high quality images and high angular resolution - is found with the orbital parameters, shown in Table 1. Such an orbit has rather fast evolution which allows to realize during the mission lifetime different geometrical configurations with networks of ground radio telescopes.

The new K4 VLBI data acquisition system based on advanced TV technology will be used to store wide-band VLBI data. Its maximal sampling rate is $128 \mathrm{Mb} / \mathrm{s}$.

To realize all VSOP scientific potential a wide collaboration with ground radio observatories is assumed. Certain plans to co-observe with VSOP are expressed by European and US VLBI networks, Australia Telescope National Facility, radio observatories in China, Russia, South Africa.

\section{Conclusion Remarks}

VLBI is a collaborative venture due to its deep inherent physical nature. This character is even emphasized in Space application. Indeed, success of so sophisticated experiment as coherent combining of data registered simultaneously at number of ground radio observatories and orbital radio telescope is possible only under highly co-ordinated efforts of thousands of experts around the whole world. Some managing decisions and creating of international body to coordinate RadioAstron and VSOP missions might become a prototype of operational agency for SVLBI network in the XXI century. Such challenges, as an operation of SVLBI in Space-Space mode and in mm wavelengths will become inevitable continuation of the current step of SVLBI. 


\section{Acknowledgements}

The authors would like to thank the international collective of many thousands of people who are working to implement Space VLBI missions. LIG thanks International Astronomical Union, and both LIG and GST thank Sydney University and Australia Telescope National Facility for financial assistance of participating in the Symposium. National Astronomy and Ionosphere Center is operated by Cornell University under contract with the National Science Foundation.

\section{References}

Alekseev V.A.: 1993, in Propagation Effects in Space VLBI, Proceedings of the Workshop, Leningrad, 1990, ed(s)., L.I.Gurvits, National Astronomy and Ionosphere Center, 123

Andreyanov V.V. et al.: 1984, Proc. of the ESA Workshop on QUASAT Project ESA SP-213,

Braginsky V.B. et al.: 1992, in Astrophysics on the Threshold of the 21st Century, ed(s)., N.S.Kardashev, Gordon \& Breach Sci. Publishers: Philadelphia, 315

Gurvits L.I., Pogrebenko S.V.: 1985, Astrophys. Space Sci. 112, 337

Gurvits L.I.: 1993, in These Proceedings, ed(s)., J.G.Robertson and W.J.Tango, Kluwer Academic Publishers: Dordrecht, in press

Conway J.E., Cornwell T.J., Wilkinson P.N.: 1990, MNRAS 246, 490

Kardashev N.S., Slysh V.I.: 1988, in The Impact of VLBI on Astrophysics and Geophysics, Proceedings of the 129th Symposium of the IAU, ed(s)., M.J.Reid and J.M.Moran, Kluwer Academic Publishers: Dordrecht, 433

Kawaguchi N.: 1991, in Frontiers of VLBI. Proceedings of the International VSOP Symposium and mm-Wave VLBI Workshop, ed(s)., H.Hirabayashi, M.Inoue, H.Kobayashi, Universal Academy Press, Inc.: Tokyo, 269

Kellermann K.I.: 1993, Nature in press,

Kellermann K.I., Pauliny-Toth I.I.K.: 1969, ApJ Lett 155, L71

Levy G.S. et al.: 1986, Science 234, 187

Nishimura 'T.: 1991, in Frontiers of VLBI. Proceedings of the International VSOP Symposium and mm-Wave VLBI Workshop, ed(s)., H.Hirabayashi, M.Inoue , H.Kobayashi, Universal Academy Press, Inc., 3

Noordam J., de Bruyn A.G.: 1982, Nature 299, 597

Pelletier G., Roland J.: 1989, Astr. Ap. 224, 24

Pilbratt G.: 1991, in Radio Interferometry: Theory, Techiques, and Applications, ed(s)., T.J.Cornwell and R.A.Perley, Astron. Soc. of the Pacific, Conf. Series v. 19, 102

Quirrenbach A. et al.: 1989, Astr. Ap. 226, L1

Reid M.J., Moran J.M., Gwinn C.R.: 1988, in The Impact of VLBI on Astrophysics and Geophysics. Proceedings of the 129th Symposium of the IAU, ed(s)., M.J.Reid and J.M.Moran, Kluwer Academic Publishers: Dordrecht, 169

Schilizzi R.T.: 1988, in The Impact of VLBI on Astrophysics and Geophysics. Proceedings of the 129th Symposium of the IAU, ed(s)., M.J.Reid and J.M.Moran, Kluwer Academic Publishers: Dordrecht, 441

Shapirovskaya N.Ya.: 1991, Sov. Astr. Lett. 17, 380

Shishov V.I.: 1993, in Propagation Effects in Space VLBI, Proccedings of the Workshop, Leningrad, 1990, ed(s)., L.I.Gurvits, National Astronomy and Ionosphere Center, 1

Slysh V.I.: 1992, $A p J$ 391, 453

Taylor A.R.: 1993, in Sub-Arcsecond Radio Astronomy, ed(s)., R.J.Davies and R.S.Booth, University Press: Cambridge, in press

Turner E.L.: 1988, in The Impact of VLBI on Astrophysics and Geophysics. Proceedings of the 129th Symposium of the IAU, ed(s)., M.J.Reid and J.M.Moran, Kluwer Academic Publishers: Dordrecht, 191

Wolszczan A., Cordes J.: 1987, A pJ Lett 320, L35

Wolszczan A., Frail D.A.: 1992, Nature 355, 145 